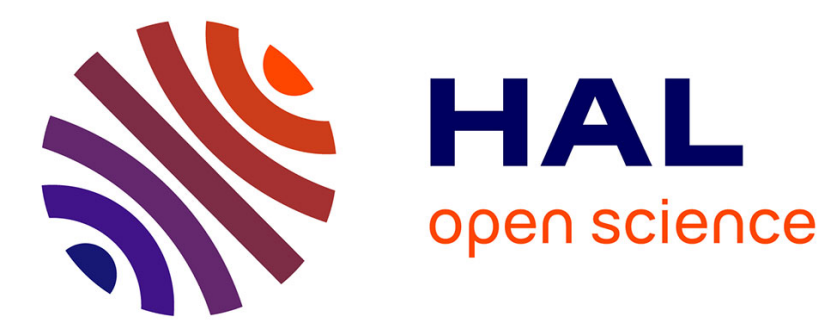

\title{
Zero temperature magnetization of the random axis chain
}

\author{
B. Derrida, A. Zippelius
}

\section{To cite this version:}

B. Derrida, A. Zippelius. Zero temperature magnetization of the random axis chain. Journal de Physique, 1986, 47 (6), pp.955-958. 10.1051/jphys:01986004706095500 . jpa-00210292

\section{HAL Id: jpa-00210292 https://hal.science/jpa-00210292}

Submitted on 1 Jan 1986

HAL is a multi-disciplinary open access archive for the deposit and dissemination of scientific research documents, whether they are published or not. The documents may come from teaching and research institutions in France or abroad, or from public or private research centers.
L'archive ouverte pluridisciplinaire HAL, est destinée au dépôt et à la diffusion de documents scientifiques de niveau recherche, publiés ou non, émanant des établissements d'enseignement et de recherche français ou étrangers, des laboratoires publics ou privés. 
Classification

Physics Abstracts

$75.10-75.30 \mathrm{~K}-75.30 \mathrm{G}-75.40 \mathrm{D}$

\title{
Zero temperature magnetization of the random axis chain
}

\author{
B. Derrida \\ Service de Physique Théorique, CEN Saclay, 91191 Gif sur Yvette, France
}

and A. Zippelius

Institut für Festkörperforschung der Kernforschungsanlage Jülich, Postfach 1913, 5170 Jülich, F.R.G.

(Reçu le 12 décembre 1985, accepté le 5 février 1986)

\begin{abstract}
Résumé. - Nous calculons l'aimantation à température nulle du modèle d'axes aléatoires à une dimension dans la limite d'une forte anisotropie et d'un faible champ magnétique $h$. Pour une distribution uniforme des axes aléatoires, nous obtenons $m=C h^{1 / \delta}$ où l'exposant critique $\delta=3$ comme pour un verre de spin d'Ising à une dimension avec une distribution gaussienne des interactions.
\end{abstract}

\begin{abstract}
We have calculated the zero temperature magnetization of the random axis chain in the limit of strong anisotropy and small magnetic field $h$. For the uniform distribution of random axes we obtain $m=C h^{1 / 8}$ with the same critical exponent $\delta=3$ as in the one dimensional Ising spinglass with a Gaussian distribution of bonds.
\end{abstract}

The random anisotropy axis model (RAM) was introduced by Harris et al. [1] to describe amorphous intermetallic compounds. The possibility of both - ferromagnetic and spinglass like behaviour - has stimulated much theoretical work. In the mean field limit the model is always ferromagnetic [2], no matter how strong the random anisotropy. Below four dimensions the ferromagnetic order is destroyed, as can be shown with a domain wall argument [3] as well as with a renormalization group analysis [4]. Whether or not the model has a spinglass phase is still a matter of controversy. Furthermore, if a spinglass phase exists, it is not clear whether its critical properties are related to those of the more conventional spinglass model of Edwards and Anderson [5].

Recently Bray and Moore [6] have studied the RAM numerically in two dimensions, using a large cell renormalization group method. They interpret their results in terms of a zero temperature phase transition in the universality class of the Ising spinglass.

The RAM in one dimension and zero applied field has been considered by Thomas [7], who showed that the ground state is nonferromagnetic. He also discussed the effect of a magnetic field in terms of domain wall argument : weak bonds of strength $\left|J_{i j}\right| \leqslant J_{0}$ divide the chain into segments of length
$L \sim 1 / J_{0}$. A magnetic field $h$ can flip the magnetic moment of such a segment $M_{L} \sim \sqrt{L}$, provided the energy gain $M_{L} h$ compensates the bond energy $J_{0}$. This yields $m=M_{L} / L=C h^{1 / 3}$.

In this note we present an exact calculation of the zero temperature magnetization for the random axis chain (RAC). The result agrees with the domain wall argument of Thomas and furthermore yields an explicit expression for the amplitude $C$.

The RAM is defined by the Hamiltonian

$$
\mathscr{H}=-J \sum_{i j}\left(\mathbf{S}_{i} \cdot \mathbf{S}_{j}\right)-D \sum_{i}\left(\mathbf{n}_{i} \cdot \mathbf{S}_{i}\right)^{2}-\mathbf{H} \cdot \sum_{i} \mathbf{S}_{i}
$$

for $d$ component spins $\mathbf{S}_{i}$ of fixed length $\mathbf{S}_{i} \cdot \mathbf{S}_{i}=1$. Nearest neighbours are coupled by a uniform exchange interaction $J>0$. The direction $\mathbf{n}_{i}\left(\mathbf{n}_{i}^{2}=1\right)$ of the local anisotropy varies randomly from site to site, whereas the strength of the anisotropy $D$ and the applied magnetic field $\mathbf{H}$ are the same for all sites. We restrict our discussion to the case of large anisotropy, $D \gg J$ and $D \gg|\mathbf{H}|$, such that the spins point either parallel or antiparallel to the local axis $\mathbf{n}_{i}$

$$
\mathbf{S}_{i}=\mathbf{n}_{i} \sigma_{i}, \quad \sigma_{i}= \pm 1
$$

In terms of these new variables the Hamiltonian 
reduces to an Ising model

$$
\mathscr{H}=-J \sum_{\langle i j\rangle}\left(\mathbf{n}_{i} \cdot \mathbf{n}_{j}\right) \sigma_{i} \sigma_{j}-\sum_{i} H_{i} \sigma_{i}
$$

with random bonds $J_{i j}=J\left(\mathbf{n}_{i} \cdot \mathbf{n}_{j}\right)$ and random fields $H_{i}=\left(\mathbf{H} \cdot \mathbf{n}_{i}\right)$. Note that the fluctuations in the bonds and in the fields are correlated.

We have calculated the groundstate energy of the model (3) in the limit of small magnetic field. Our method of calculation is a generalization to the RAC of a method already used for the onedimensional spinglass [8]. Hence we keep our discussion brief and refer the reader to reference [8] for any details.

One first derives a recursion relation for the groundstate energy of a chain with $L$ spins. If the spin $\sigma_{L}=$ $+1\left(\sigma_{L}=-1\right)$ then its groundstate energy is denoted by $-F_{L}\left(-G_{L}\right)$. These quantities obey the following coupled recursion relations

$$
\begin{aligned}
& F_{L+1}=J h_{L+1}+\max \left(F_{L}+J_{L+1, L} ; G_{L}-J_{L+1, L}\right) \\
& G_{L+1}=-J h_{L+1}+\max \left(F_{L}-J_{L+1, L} ; G_{L}+J_{L+1, L}\right)
\end{aligned}
$$

with

$$
J_{L, L+1}=J\left(\mathbf{n}_{L} \cdot \mathbf{n}_{L+1}\right) \quad \text { and } \quad h_{L}=\left(\mathbf{H} \cdot \mathbf{n}_{L}\right) / J .
$$

A single, closed equation is obtained for the difference $2 C_{L}=\left(G_{L}-F_{L}\right) / J$

$$
\begin{aligned}
C_{L+1}=C_{L}-h_{L+1} & +\max \left(-C_{L}-J_{L, L+1} / J ; 0\right)- \\
& -\max \left(C_{L}-J_{L+1, L} / J ; 0\right)
\end{aligned}
$$

$C_{L}$ remains finite as $L \rightarrow \infty$ and obeys a stationary probability distribution $P\left(C_{L}, \mathbf{n}_{L}\right)$ which depends on the local random axis $n_{L}$. It satisfies the integral equation :

$$
\begin{array}{r}
P(C, \mathbf{n})=\int \mathrm{d} C^{\prime} \int \mathrm{d} n^{\prime} \rho\left(\mathbf{n}^{\prime}\right) P\left(C^{\prime}, \mathbf{n}^{\prime}\right) \delta\left(C-C^{\prime}+(\mathbf{h} \cdot \mathbf{n})\right. \\
\left.-\max \left(-C^{\prime}-\left(\mathbf{n} \cdot \mathbf{n}^{\prime}\right) ; 0\right)+\max \left(C^{\prime}-\left(\mathbf{n} \cdot \mathbf{n}^{\prime}\right) ; 0\right)\right)
\end{array}
$$

with $\mathbf{h}=\mathbf{H} / J$.

Here we have assumed that the $\mathbf{n}_{\boldsymbol{i}}$ are independent variables, which all have the same distribution $\rho(\mathbf{n})$. Once the distribution $P(C, \mathbf{n})$ is known, one can calculate the groundstate energy $-E$, via

$$
\begin{aligned}
E=\lim _{L \rightarrow \infty} \frac{F_{L}}{L}=\lim _{L \rightarrow \infty}\left\langle F_{L+1}-F_{L}\right\rangle=2 J \int \mathrm{dn} \rho(\mathbf{n}) \int \mathrm{d} \mathbf{n}^{\prime} \rho\left(\mathbf{n}^{\prime}\right) \int \mathrm{d} C^{\prime} \times \\
\\
P\left(C^{\prime}, \mathbf{n}^{\prime}\right)\left[\max \left(0 ; C^{\prime}-\left(\mathbf{n} \cdot \mathbf{n}^{\prime}\right)\right)+\left(\mathbf{n} \cdot \mathbf{n}^{\prime}\right)+(\mathbf{h} \cdot \mathbf{n}) / 2\right]
\end{aligned}
$$

where the average $\langle\ldots\rangle$ is taken over $\mathbf{n}, \mathbf{n}^{\prime}$ and $C^{\prime}$.

In this section we present the solution of the integral equation for $P(C, \mathrm{n})$. As a first step we break up the integration over $C^{\prime}$ and work out the various contributions explicitly :

$$
\begin{aligned}
P(C-\mathbf{h} \cdot \mathbf{n}, \mathbf{n})=\int \mathrm{d} \mathbf{n}^{\prime} \rho\left(\mathbf{n}^{\prime}\right) & {\left[\int_{|C|}^{\infty} \mathrm{d} C^{\prime} \delta\left(C-\left(\mathbf{n} \cdot \mathbf{n}^{\prime}\right)\right)+\int_{-\infty}^{-|C|} \mathrm{d} C^{\prime} \delta\left(C+\left(\mathbf{n} \cdot \mathbf{n}^{\prime}\right)\right)\right] P\left(C^{\prime}, \mathbf{n}^{\prime}\right)+} \\
+ & \int \mathrm{dn}^{\prime} \rho\left(\mathbf{n}^{\prime}\right)\left[P\left(C, \mathbf{n}^{\prime}\right) \theta\left(\mathbf{n} \cdot \mathbf{n}^{\prime}-|C|\right)+P\left(-C, \mathbf{n}^{\prime}\right) \theta\left(-\left(\mathbf{n} \cdot \mathbf{n}^{\prime}\right)-|C|\right)\right] .
\end{aligned}
$$

To solve this equation, it is useful to look at the moments of the distribution function

$$
Q_{m}(C, h)=\int \operatorname{dn} \rho(\mathbf{n})(\mathbf{h} \cdot \mathbf{n})^{m} P(C, \mathbf{n}) .
$$

In the following we specialize to the isotropic distribution $\rho(\mathbf{n})$ and consider the limit of a weak magnetic field only. In that case the angular dependence of the integral kernel (9) allows one to derive a closed set of equations for the low order moments. For zero magnetic field one has the solution $Q_{0}(C, h=0)=\delta(C)$. For finite but small magnetic field we expect $Q_{0}(C, h)$ to be concentrated in a small region around $C=0$. We multiply equation (9) by $(\mathbf{h} \cdot \mathbf{n})^{m}$, integrate over $\mathbf{n}$ and expand for small $C$. The first three equation $(m=0,1,2)$ read :

$$
\begin{gathered}
\int \mathrm{d} \mathbf{n} P(C-\mathbf{h} \cdot \mathbf{n}, \mathbf{n})=A_{d} \int_{|C|}^{\infty} \mathrm{d} C^{\prime}\left(Q_{0}\left(C^{\prime}, h\right)+Q_{0}\left(-C^{\prime}, h\right)\right)+\frac{1}{2}\left(Q_{0}(C, h)+Q_{0}(-C, h)\right) \\
\int \mathrm{d} \mathbf{n} P(C-\mathbf{h} \cdot \mathbf{n}, \mathbf{n})(\mathbf{h} \cdot \mathbf{n})=C A_{d} \int_{|C|}^{\infty} \mathrm{d} C^{\prime}\left(Q_{1}\left(C^{\prime}, h\right)-Q_{1}\left(-C_{1}^{\prime}, h\right)\right)+\frac{A_{d}}{d-1}\left(Q_{1}(C, h)-Q_{1}(-C, h)\right)
\end{gathered}
$$




$$
\begin{aligned}
\int \mathrm{d} \mathbf{n}(C-\mathbf{h} \cdot \mathbf{n}, \mathbf{n})(\mathbf{h} \cdot \mathbf{n})^{2} & =h^{2} \frac{A_{d}}{d-1} \int_{|C|}^{\infty} \mathrm{d} C^{\prime}\left(Q_{0}\left(C^{\prime}, h\right)+Q_{0}\left(-C^{\prime}, h\right)\right) \\
& -\frac{A_{d}}{d-1} \int_{|C|}^{\infty} \mathrm{d} C^{\prime}\left(Q_{2}\left(C^{\prime}, h\right)+Q_{2}\left(-C^{\prime}, h\right)\right)+\frac{h^{2}}{2 d}\left(Q_{0}(C, h)+Q_{0}(-C, h)\right) \\
+ & \frac{A_{d}}{d-1}|C|\left(Q_{2}(C, h)+Q_{2}(-C, h)\right)
\end{aligned}
$$

with

$$
A_{d}=\Gamma(d / 2) /\left(\sqrt{\pi} \Gamma\left(\frac{d-1}{2}\right)\right)
$$

Let us look for a solution which has the following properties :

a) $Q_{0}$ and $Q_{2}$ are even in $C$ and $Q_{1}$ is odd in $C$.

b) For small $C$ and $h, Q_{m}(C, h)$ have a scaling form

$$
\begin{aligned}
& Q_{0}(C, h)=h^{\alpha_{0}} F_{0}\left(h^{\beta} C\right) \\
& Q_{1}(C, h)=h^{\alpha_{1}} F_{1}\left(h^{\beta} C\right) \\
& Q_{2}(C, h)=h^{\alpha_{2}} F_{2}\left(h^{\beta} C\right) .
\end{aligned}
$$

We shall use this ansatz to simplify the equations and then show explicitly that this ansatz does indeed solve the equations.

To leading order in $C$ and $h$ equations $(12,13)$ imply

$$
h^{\alpha_{2}} F_{2}(x)=\frac{1}{d} h^{2+\alpha_{0}} F_{0}(x)
$$

and

$$
\left(1-2 \frac{A_{d}}{d-1}\right) F_{1}(x)=h^{\alpha_{2}-\alpha_{1}+\beta} F_{2}^{\prime}(x) .
$$

Therefore our ansatz is only consistent if we choose

$$
\begin{aligned}
\alpha_{2} & =2+\alpha_{0} \\
\alpha_{2} & =\alpha_{1}-\beta \\
F_{2} & =\frac{1}{d} F_{0}
\end{aligned}
$$

and

$$
F_{1}=F_{2}^{\prime}(d-1) /\left(d-1-2 A_{d}\right) .
$$

With these results, equation (11) reads

$$
-B h^{2+3 \beta} F_{0}^{\prime \prime}(x)=\int_{x}^{\infty} F_{0}(y) \mathrm{d} y-x F_{0}(x)
$$

where

$$
B=\left(2 A_{d}+d-1\right) /\left(4 A_{d} d\left(d-1-2 A_{d}\right)\right) .
$$

Hence one must have

$$
\beta=-\frac{2}{3} .
$$

With the substitution

$$
\varphi(x)=\int_{x}^{\infty} \mathrm{d} y F_{0}(y)
$$

we can reduce equation (18) to an ordinary differential equation

$$
B \varphi^{\prime \prime \prime}(x)=\frac{\mathrm{d}}{\mathrm{d} x}(x \varphi(x))
$$

This equation is solved by

$$
\varphi(x)=\Psi\left(B^{-1 / 3} x\right) / \Psi(0)
$$

with

$$
\Psi(x)=\sqrt{x} K_{1 / 3}\left(\frac{2}{3} x^{3 / 2}\right)
$$

and $K_{1 / 3}$ a Bessel function.

The groundstate energy is a weighted integral of the stationary probability distribution (Eq. (8)). In particular for the isotropic distribution $\rho(\mathbf{n})$, it is given by

$$
\begin{array}{r}
E=2 J \int \mathrm{d} \mathbf{n} \rho(\mathbf{n}) \int \mathrm{d} \mathbf{n}^{\prime} \rho\left(\mathbf{n}^{\prime}\right) \int \mathrm{d} C^{\prime} P\left(C^{\prime}, \mathbf{n}^{\prime}\right) \times \\
\times \max \left(0 ; C^{\prime}-\mathbf{n} \cdot \mathbf{n}^{\prime}\right) .
\end{array}
$$

For small $h$ we have seen, that $P(C, \mathbf{n})$ is concentrated in a small region around $C=0$. Hence we can expand around $C=0$ and obtain

$$
\frac{E}{J}=\frac{2 A_{d}}{d-1}+A_{d} \frac{\int \mathrm{d} C C^{2} Q_{0}(C, h)}{\int \mathrm{d} C Q_{0}(C, h)} .
$$

This integral can be further transformed with help of 
the substitutions as defined above

$$
\begin{aligned}
\Delta E / J=(E(h) & -E(0)) / J= \\
& =2 A_{d} h^{-2 \beta} \int_{0}^{\infty} \mathrm{d} x x \varphi(x) / \varphi(0) \\
& =2 A_{d} \frac{B^{2 / 3}}{h^{2 \beta}} \int_{0}^{\infty} \mathrm{d} x x \Psi(x) / \Psi(0) \\
& =2 A_{d} 3^{1 / 3} \frac{\Gamma(2 / 3)}{\Gamma(1 / 3)} B^{2 / 3} h^{4 / 3}
\end{aligned}
$$

We differentiate with respect to $H$, to obtain our final result for the magnetization

$$
\begin{aligned}
m(H)=\frac{\mathrm{d} E}{\mathrm{~d} H}=\left(\frac{H}{J}\right)^{1 / 3} 8 A_{d} \frac{\Gamma(2 / 3)}{\Gamma(1 / 3)} \times \\
\\
\times\left(\frac{2 A_{d}+d-1}{12 d A_{d}\left(d-1-2 A_{d}\right)}\right)^{2 / 3}
\end{aligned}
$$

where $A_{d}$ is given in equation (14). This implies $m \simeq 0.9050(H / J)^{1 / 3}$ for $x y$ spins $(d=2)$ and $m \simeq 0.6123(H / J)^{1 / 3}$ for Heisenberg spins $(d=3)$.
To conclude : the exact calculation agrees with the result $-m=C h^{1 / 3}-$ of a domain wall argument and explicitely yields the amplitude $C$. The critical exponent $\delta$ is the same as for the onedimensional Ising spin glass with a nonzero probability of zero bonds [8]. A domain wall argument exists also for the Ising spin glass [9] with an arbitrary distribution of bonds. In that case the exponent $\delta$ was shown to depend on the density of bonds at $J_{i j}=0[8,9]$. It would certainly be interesting to generalize our calculation for the RAC to an arbitrary distribution of random axes [10]. Our method of solution, using the moments $Q_{m}$ of the distribution function $P(C, \mathrm{n})$, relies on the isotropy of $\rho(\mathbf{n})$. So far we have not been able to generalize it to an arbitrary distribution $\rho(\mathbf{n})$.

\section{Acknowledgments.}

One of us (A.Z.) would like to thank P. Rujan for a very useful discussion and the CEN Saclay for its hospitality.

\section{References}

[1] Harris, R., Plischke, M. and Zuckermann, M., Phys. Rev. Lett. 31 (1973) 160.

[2] Derrida, B. and Vannimenus, J., J. Phys. C 13 (1980) 3261.

[3] Jayaprakash, C. and KirkPatrick, S., Phys. Rev. B 21 (1980) 4072.

[4] Aharony, A., Phys. Rev. B 12 (1975) 1038.

[5] Edwards, S. F. and ANDERSon, P. W., J. Phys. F 5 (1975) 1965.
[6] Bray, A. and Moore, M., J. Phys. C 18 (1985) L139. [7] Thomas, H., in Ordering in Strongly Fluctuating Condensed Matter Systems, Ed. T. Riste (Plenum, New York) 1980.

[8] Gardner, E. and Derrida, B., J. Stat. Phys. 39 (1985) 367.

[9] Chen, H. H. and MA, S. K., J. Stat. Phys. 29 (1982) 717. [10] Fischer, K. H. and Zippelius, A., J. Phys. C 18 (1985) L1139. 